\title{
Comparative experimental and theoretical study on electron scattering by propane
}

\author{
W. Y. Baek, T. Braunroth, M. U. Bug, F. Heimbach, H. Nettelbeck, and H. Rabus \\ Physikalisch-Technische Bundesanstalt (PTB), Bundesallee 100, 38116 Braunschweig, Germany
}

(Received 8 April 2019; published 25 July 2019)

\begin{abstract}
Combined experimental and theoretical studies on electron scattering by propane were performed over a wide energy and angular range. The experimental values were compared to the results calculated using currently available theoretical and semiempirical approaches. The widely used independent atom model with screening corrected additivity rule coarsely reproduces the angular-dependent differential elastic cross sections of propane in the measured energy between $20 \mathrm{eV}$ and $1 \mathrm{keV}$. For electron energies above $40 \mathrm{eV}$, the modified independent atom model, which takes into account multiple scattering within the molecule, yields better agreement with the experimental results. Total, integral elastic and integral inelastic electron scattering cross sections of propane were calculated using the spherical complex optical potential model and the variable phase approach. The suitability of the theoretical model is discussed by means of the experimental data.
\end{abstract}

DOI: 10.1103/PhysRevA.100.012710

\section{INTRODUCTION}

For many decades, electron collision cross sections of hydrocarbons have been a subject of continual interest due to their importance in many areas of applied science and technology. Electron collisions with hydrocarbons play a dominant role in plasma generation sources and in the boundary region of magnetic fusion devices such as the International Thermonuclear Experimental Reactor (ITER) [1]. In the latter, a variety of hydrocarbon molecules are produced by the interaction of the hydrogen plasma with carbon-clad plasma facing materials. Light hydrocarbon molecules are also of importance for radiation dosimetry. They are frequently used as filling gases in radiation detectors which are employed to simulate energy deposition by ionizing radiation in tissue on cellular and subcellular levels [2,3].

Comprehensive electron scattering cross sections of hydrocarbon molecules are required for the modeling of plasma conditions and for the characterization of tissue equivalent radiation detectors. However, there exist only fragments of such cross section data in the literature, despite the numerous experimental and theoretical studies on electron-molecule collisions that have been carried out. This work aims to address this issue by focusing on the total electron scattering (TCS) and differential elastic scattering (DCS) cross sections of propane $\left(\mathrm{C}_{3} \mathrm{H}_{8}\right)$. Doubly differential ionization cross sections of propane have been recently published by the present authors [4]. Experimental TCS of propane have been reported by several groups [5-9] for electron energies below $1 \mathrm{eV}$ up to $4 \mathrm{keV}$. For the DCS of propane, only two literature sources [10,11] provide numerical values. Boesten et al. [10] measured the DCS of propane for electron energies from 2 to $100 \mathrm{eV}$ over scattering angles ranging from $10^{\circ}$ to $130^{\circ}$. De Souza et al. [11] reported the DCS of propane over a similar angular range, but their measurements extended to higher energies that ranged from 40 to $500 \mathrm{eV}$.

In the present work, the DCS $d \sigma_{e l} / d \Omega$ of propane were measured absolutely for primary electron energies $T$ between
$20 \mathrm{eV}$ and $1 \mathrm{keV}$ in the range of scattering angles $\theta$ from $10^{\circ}$ to $135^{\circ}$. This work also includes TCS $\sigma_{t}$ of propane which were determined for primary electron energies between about $3 \mathrm{eV}$ and $2 \mathrm{keV}$ within the framework of the European project called NANDET. The original data of these TCS have been published (in tabular form) in the scientific report of the project [12]; however, there is not an adequate description of the experimental procedure and discussion of the results.

Differential elastic scattering cross sections of propane were also calculated using the independent atom model with screening corrected additivity rule (IAM-SCAR) [13] as well as the modified independent atom model (MIAM) [14]. Although the MIAM method has proved to be a suitable theoretical approach for the calculation of DCS of polyatomic molecules for $T>50 \mathrm{eV}[15,16]$, it has rarely been used since its publication in the 1970s. Instead, the IAM-SCAR method has become the predominant approach for the calculation of DCS of molecules for electron energies down to almost $20 \mathrm{eV}$ [17-24]. The reason for this may be the simplicity of the IAM-SCAR method in contrast to the complex, rather computing-intensive MIAM method. In this work, both methods have been evaluated using the present experimental DCS as benchmark data. In addition, integral elastic, inelastic, and total electron scattering cross sections were calculated by means of the spherical complex optical potential (SCOP) method [25-29] and compared to the present experimental results as well as to the integral ionization cross sections of propane recently published by Baek et al. [4].

\section{EXPERIMENT}

Total electron scattering cross sections $\sigma_{t}$ were measured by means of a linear transmission experiment based on the Lambert-Beer attenuation law:

$$
I=I_{0} \exp \left(-\sigma_{t} n L\right)
$$

where $I_{0}$ is the initial current of the electron beam and $I$ is the attenuated beam current after passing a gas layer with 
number density $n$ and length $L$. A detailed description of the experimental setup can be found in a previous paper by Baek and Grosswendt [30]. The main components of the apparatus are an electron gun, a scattering chamber and an electron energy analyzer. The electron gun [31] consisted of a tungsten filament cathode, a focusing lens, and two orthogonal pairs of deflection plates. It delivered a well-collimated beam for energies between $20 \mathrm{eV}$ and $5 \mathrm{keV}$. The energy width of the electron beam was about $0.5 \mathrm{eV}$.

Electrons underwent collision processes with the molecules of interest in the scattering chamber that was connected to the gas source via a regulating valve. The electron beam entered and exited the scattering chamber through an entrance and exit aperture, both $0.5 \mathrm{~mm}$ in diameter. The length of the scattering chamber was $132 \mathrm{~mm}$. Electrons leaving the scattering chamber were analyzed with respect to their energy by means of a hemispherical electron energy analyzer with a mean radius of $100 \mathrm{~mm}$ and a deflection angle of $150^{\circ}$. A channel electron multiplier was used as the detector. The electron energy analyzer was used to discriminate against the electrons that were scattered in the forward direction following collision processes with molecules in the scattering chamber. The distance between the exit aperture of the scattering chamber and the entrance slit of the hemispherical condenser was $318 \mathrm{~mm}$, leading to a solid angle of $7.1 \times 10^{-4} \mathrm{sr}$. The pass energy of the electron energy analyzer was set equal to the primary electron energy so that electrons which lost energy during collision processes were unable to reach the detector located at the end of the analyzer. The energy resolution of the energy analyzer was $1 \mathrm{eV}$ full width at half maximum (FWHM) for 2-keV electrons and was better at lower energies. It should be noted that this resolution is insufficient to resolve the energy loss due to rotational and vibrational excitations. In order to avoid the deflection of electrons due to the Earth's magnetic field, the entire electron beam path was surrounded by $\mu$-metal cylinders.

As the electron gun delivered stable beams only for energies above $20 \mathrm{eV}$, the measurement of TCS below $20 \mathrm{eV}$ was performed using a $20-\mathrm{eV}$ electron beam together with a retarding voltage $U_{R}$ applied to the scattering chamber [30]. The energy of the electron beam was reduced by $e U_{R}$ at the entrance aperture of the scattering chamber so that the electron-molecule collision processes took place at a reduced energy of $20 \mathrm{eV}-e U_{R}$, where $e$ is the electron charge. The pass energy of the electron energy analyzer remained fixed at $20 \mathrm{eV}$ since electrons leaving the scattering chamber were accelerated to the initial energy on the way to the electron energy analyzer, which was at Earth potential.

Total electron scattering cross sections were measured by recording the detector count rate as a function of the gas pressure in the scattering chamber. The gas pressure, which was set by means of a regulating valve with an adjustable leak rate between $10^{-9}$ and $600 \mathrm{mbarl} / \mathrm{s}$, was measured with a capacitance manometer whose reading was corrected for the thermal transpiration effect [32]. The maximal gas pressure was chosen such that the beam attenuation did not exceed $90 \%$. Gas streaming out through the apertures of the scattering chamber was removed by a turbomolecular pump with a pumping speed of $2000 \mathrm{l} / \mathrm{s}$. The initial electron current was chosen such that the counting efficiency of the channel electron multiplier was almost independent of the count rate.

The experimental method for the determination of DCS has been described in detail in earlier works of Baek et al. [4,16]. In brief, the angle-dependent count rate of electrons scattered elastically was converted into $d \sigma_{e l} / d \Omega$ via

$$
\frac{d \sigma_{e l}}{d \Omega}(\theta, T)=\frac{1}{V_{\mathrm{eff}} \eta(T)} \frac{e}{I_{0}} \frac{\Delta \dot{N}_{e l}}{\Delta \Omega}(\theta, T),
$$

where $V_{\text {eff }}$ is the effective scattering volume [33] and $\eta$ is the detection efficiency of the electron energy analyzer. The former was determined from the current loss of the primary electron beam after its passage through the interaction zone and the TCS of propane at the respective energy. The detection efficiency was obtained from acknowledgment of the fact that electron scattering of helium below the ionization threshold is almost entirely elastic in nature. In this case, $\eta$ can be determined from the ratio of the total detector count rate of scattered electrons to the current loss of the primary electron beam. The dependence of $\eta$ on $T$ was determined by simulating the electron trajectory from the interaction zone to the detector. The accuracy of the simulation was checked by the comparison of the theoretical and experimental elastic peak shapes [16].

The measurement of the DCS was carried out with the same apparatus used for the determination of ionization cross sections described in Ref. [4]. An effusive gas source consisting of a cylindrical tube was used for the generation of the molecular beam. The gas pressure on the top of the gas nozzle was about 0.5 mbar, leading to a number of molecules per area of $5 \times 10^{13} \mathrm{~cm}^{-2}$ in the interaction zone. The gas pressure was adjusted such that the single collision condition was fulfilled. The electron gun was of the same type as that employed for the determination of TCS. The electron current varied between $1.0 \mathrm{pA}$ at $T=20 \mathrm{eV}$ and $1.0 \mathrm{nA}$ at $T=$ $1 \mathrm{keV}$. With this current, the statistical uncertainty of counts in the elastic peak could be kept below $3 \%$ at a collection time of 3 min. Higher electron beam current was not applied to avoid the change of the detector gain at high count rates, which may arise due to saturation effect caused by space charge in the channel electron multiplier.

Electron energies were analyzed using a hemispherical spectrometer with a mean radius of $150 \mathrm{~mm}$ and a deflection angle of $180^{\circ}$. Scattering angles were adjusted by rotating the electron gun relative to the electron energy analyzer. Angular resolution of the experiment could be adjusted using an iris aperture located between the interaction zone and entrance aperture to the hemispherical deflector. The full acceptance angle of the electron energy analyzer was set to $3^{\circ}$ for scattering angles above $35^{\circ}$ and $1.6^{\circ}$ for scattering angles below $35^{\circ}$. Its energy resolution (FWHM) was $1.7 \mathrm{eV}$ for $T=$ $1 \mathrm{keV}$ and was better at lower energies. As in the case of the measurement of the TCS, this energy resolution is insufficient to resolve between elastic scattering and rotational and vibrational excitations. The elastic count rate $\Delta \dot{N}_{e l}$ in Eq. (2) was obtained from the area of the elastic peak in the energy spectrum of secondary electrons generated by recording the detector count rate as a function of the analyzer pass energy. 
Prior to obtaining this count rate, the background spectrum arising from electrons scattered outside the interaction zone was subtracted. This background spectrum was measured by injecting gas molecules diffusively through a hole in the wall of the scattering chamber. The injection rate was adjusted such that the residual gas pressure in the main and background measurement was equal.

\section{THEORY AND CALCULATION}

At electron energies higher than $1 \mathrm{keV}$, the independent atom model (IAM) [34,35] is a reasonable approximation for the calculation of the DCS of polyatomic molecules. In this approximation, the change of the charge distribution due to molecular bindings is neglected and each atomic constituent of the molecule is regarded as an independent scattering center. The molecular scattering amplitude can then be represented as the sum of the scattering amplitudes of the atomic constituents. When averaged over all orientations of the molecular axes, the DCS of a molecule in the IAM is given by

$$
\frac{d \sigma_{e l}}{d \Omega}(\theta)=\sum_{A=1}^{N}\left|f_{A}(\theta)\right|^{2}+\sum_{A \neq B}^{N} f_{A}^{*}(\theta) f_{B}(\theta) \frac{\sin q r_{A B}}{q r_{A B}},
$$

where $f_{A}(\theta)$ is the scattering amplitude of the atom $A$ in the molecule, $N$ is the number of atoms in the molecule, and $q$ is the momentum transfer and $r_{A B}$ is the distance between the atoms $A$ and $B$. The scattering amplitudes of atoms, in this case hydrogen and carbon, were calculated using the partial wave expansion method:

$$
f_{A}(\theta)=\frac{1}{2 i k} \sum_{l}(2 l+1)\left[\exp \left(2 i \delta_{l}^{(A)}\right)-1\right] P_{l}(\cos \theta),
$$

where $\delta_{l}^{(A)}$ is the phase shift of the $l$ th partial wave due to atom $A$ and $P_{l}(x)$ is a Legendre polynomial. The phase shifts $\delta_{l}^{(A)}$ were calculated by solving the radial Schrödinger equation,

$$
\left[\frac{d^{2}}{d r^{2}}+k^{2}-\frac{l(l+1)}{r^{2}}-2 V_{A}(r)\right] \varphi_{l}=0,
$$

where $V_{A}(r)$ is the interaction potential of the atom $A$ for electrons consisting of an electrostatic and exchange potential. Equation (5) was solved using the numerical algorithms implemented in the program ELSEPA published by Salvat et al. [36]. In the present work, the Dirac-Hartree-Fock electron density model was employed to determine atomic electrostatic potentials. The exchange potential was calculated using the semiempirical model of Furness and McCarthy [37].

The approximation that each atomic constituent acts as an independent scattering center is applicable as long as the wavelength of incident electrons is much shorter than the typical bond lengths in the molecule. At low and intermediate electron energies, where the electron wavelength is comparable to the molecular bond lengths, the IAM method overestimates the DCS of molecules. There exists to date two established IAM-based models, the IAM-SCAR model and the MIAM, both of which provide satisfactory results also at low electron energies. In the IAM-SCAR model, which was derived on the basis of geometrical considerations $[17,18]$, the
DCS of a molecule consists of a direct and redispersed term. The direct term is given by Eq. (3) with the modification that the scattering amplitudes $f_{A}(\theta)$ of the atomic constituents are multiplied by screening coefficients. These coefficients take into account the decrease of electron flux due to geometrical overlapping of the atoms within the molecule. The formulas for the calculation of the screening coefficients and of the redispersed term can be found in Ref. [18].

In contrast to the IAM-SCAR model, the MIAM is an $a b$ initio approach derived by expanding the $T$ matrix as a series of multiple scattering terms. In this approach, the orientation-averaged DCS of a molecule owing to a shortrange potential is written as

$$
\frac{d \sigma_{e l}}{d \Omega}(\theta, T)=I_{S}+I_{S S}+I_{S D}^{(1)}+I_{S D}^{(2)}+I_{D D}^{(0)}+\cdots,
$$

where $I_{S}$ and $I_{S S}$ are equal to the first and second sum on the right-hand side in Eq. (3), respectively. Other remaining terms on the right-hand side in Eq. (6) describe multiple scattering of the incident electron wave inside the molecule. The equations for the multiple scattering terms can be found in Ref. [14].

In this work, the DCS of propane were calculated using both of the aforementioned models. The TCS $\sigma_{t, A}$ of the atomic constituent required for the determination of the screening coefficients in the IAM-SCAR model was obtained using the optical theorem [38]:

$$
\sigma_{t, A}=\frac{4 \pi}{k^{2}} \operatorname{Im}\left[f_{A}(\theta=0)\right] .
$$

The DCS with the MIAM approach were calculated also using the phase shifts and the scattering amplitudes determined by means of Eqs. (4) and (5). It should be noted that the off-shell integral $Q$ appearing in the multiple scattering terms in Eq. (6) was determined using the pole approximation [14], in contrast to earlier works of Baek et al. [16] and other authors $[15,39]$, where $Q$ was set to zero. While the pole approximation provides a rather simple analytical expression for $Q$, it has the disadvantage that it diverges at small momentum transfers and high numbers of $l$. Therefore, the multiple scattering terms $I_{S D}^{(1)} I_{S D}^{(2)}$, and $I_{D D}^{(0)}$ were calculated with a limited number of partial waves. The maximum number $l_{\max }$ of partial waves for the multiple scattering terms was 20 for $T=1 \mathrm{keV}$ and at lower energies, this maximum was smaller. The geometry of propane, which is required in both methods, was obtained by means of the Hartree-Fock geometry optimization with the software GAUSSIAN09 [40] and the $\mathrm{HF} / 6-311 \mathrm{G}^{* *}$ basis sets.

In addition to the DCS, integral elastic cross sections, integral inelastic cross sections, and TCS of propane were theoretically determined by means of the SCOP model [25-29]. In the fixed-nuclei approximation, the electron-molecule interaction can be described by a complex optical potential $V_{\text {opt }}(\vec{r})$ consisting of a real and imaginary part:

$$
\begin{aligned}
V_{o p t}(\vec{r}) & =V_{R}(\vec{r})+i V_{\mathrm{abs}}(\vec{r}) \\
& =V_{s t}(\vec{r})+V_{e x}(\vec{r})+V_{c p}(\vec{r})+i V_{\mathrm{abs}}(\vec{r}) .
\end{aligned}
$$

The real part $V_{R}(\vec{r})$ represents elastic scattering. It is the sum of electrostatic potential $V_{s t}(\vec{r})$, exchange potential $V_{e x}(\vec{r})$, and correlation-polarization potential $V_{c p}(\vec{r})$. The imaginary 
part $V_{\text {abs }}(\vec{r})$ accounts for the absorption of the incident waves into inelastic channels such as excitation and ionization.

The potentials specified in Eq. (8) depend on the electron density distribution $\rho(\vec{r})$ in the molecule. Its calculation requires the molecular orbital wave functions, which were obtained by means of the software GAUSSIAN09 [40] deploying the $\mathrm{HF} / 6-311 \mathrm{G}^{* *}$ basis sets. The exchange potential $V_{e x}(\vec{r})$ was then calculated using the Hara free-electron-gas exchange (HFEGE) model [41] and the correlation-polarization potential $V_{c p}(\vec{r})$ was obtained with the modified free-electron gas (FEG) model [42,43] using asymptotic corrections [44].

The absorption potential $V_{\text {abs }}(\vec{r})$ was determined by means of the quasifree-scattering model proposed by Staszewska et al. [45]:

$$
V_{\mathrm{abs}}(\vec{r})=-\rho(\vec{r}) \sqrt{\frac{T_{\mathrm{loc}}}{2}} \bar{\sigma}_{b}(\rho(\vec{r}), k),
$$

where the local kinetic energy $T_{\text {loc }}$ is defined as

$$
T_{\mathrm{loc}}=T-V_{s t}(\vec{r})-V_{e x}(\vec{r}) .
$$

The inelastic binary collision cross section $\bar{\sigma}_{b}$ is given by

$$
\bar{\sigma}_{b}=\frac{8 \pi}{5 k^{2} k_{F}^{3}} H\left(k^{2}-k_{F}^{2}-2 \Delta\right)\left(A_{1}+A_{2}+A_{3}\right),
$$

with $k_{F}=\left[3 \pi^{2} \rho(\vec{r})\right]^{1 / 3}$ and

$$
\begin{aligned}
& A_{1}=\frac{5 k_{F}^{3}}{2 \Delta}, \quad A_{2}=-\frac{k_{F}^{3}\left(5 k^{2}-3 k_{F}^{3}\right)}{\left(k^{2}-k_{F}^{2}\right)^{2}}, \\
& A_{3}=2 H\left(2 k_{F}^{2}+2 \Delta-k^{2}\right) \frac{\left(2 k_{F}^{2}+2 \Delta-k^{2}\right)^{2}}{\left(k^{2}-k_{F}^{2}\right)^{2}} .
\end{aligned}
$$

In Eqs. (11) and (12), $H(x)$ is the Heaviside step function, such that $H(x)=1$ for $x \geqslant 0$ and $H(x)=0$ for $x<0$. Disregarding rotational, vibrational, and electronic excitations, $\Delta$ is set to $10.94 \mathrm{eV}$ which is the ionization potential of propane [46].

The electron density distribution in polyatomic molecules is usually anisotropic, which is associated also with anisotropic interaction potentials. One way to solve a scattering problem with anisotropic interaction potentials is the multipole expansion of the potentials around the center of mass of the molecule [44]. This leads to a factorization of the potentials in radial and angular components:

$$
V_{o p t}^{p \mu}(\vec{r})=\sum_{l, h} v_{l h}(r) X_{l h}^{(p \mu)}(\theta, \phi),
$$

where $p \mu$ denotes one of the irreducible representations of the molecular point group and one of its components, respectively. The symmetry adapted function $X_{l h}^{(p \mu)}$ is a linear combination of real spherical harmonics $S_{l m}$ :

$$
X_{l h}^{(p \mu)}=\sum_{m=-l}^{l} b_{h l m}^{(p \mu)} S_{l m}(\theta, \phi),
$$

where the coefficients $b_{h l m}^{(p \mu)}$ can be obtained from the character tables of the irreducible representations. For closed-shell nonlinear molecules in their ground state, $p \mu$ is the totally symmetric $A_{1}$ irreducible representation and $h=1$. In this work,

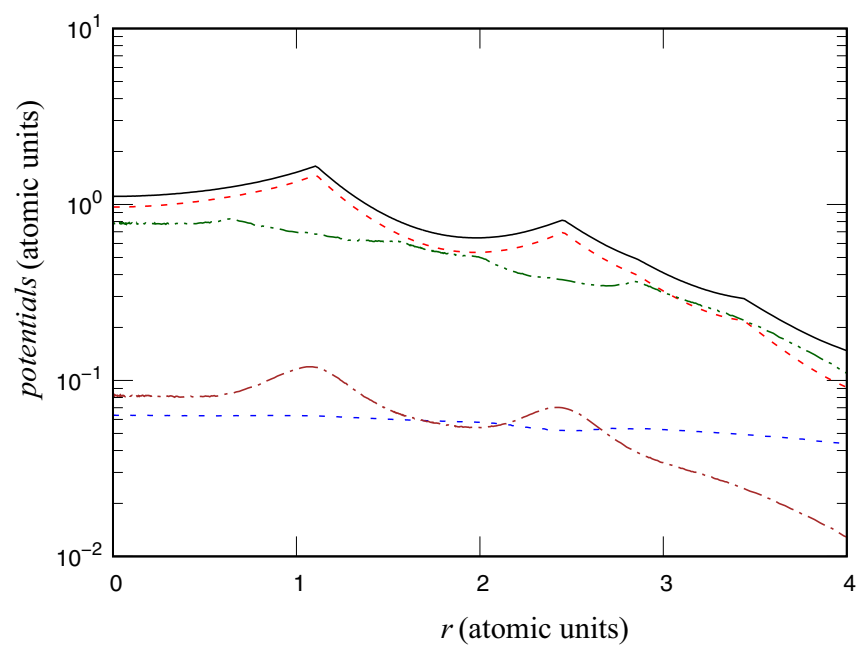

FIG. 1. Optical potential of propane for $T=100 \mathrm{eV}$ as function of the distance $r$ from the center of mass of the molecule: $(---)$ $V_{s t},(\cdots) V_{c p},(-\cdots-) V_{e x},(-) V_{R},(-\cdot-) V_{\mathrm{abs}}$.

the multipole expansion of the potentials specified in Eq. (8) was performed with the numerical algorithms implemented in SCELIB3.0 [44].

The main feature of the SCOP model is that only the isotropic term $l=0$ is retained in the multipole expansion. In other words, only the term $v_{01}$ in Eq. (13), and $b_{010}$ and $S_{00}$ in Eq. (14) are of relevance. It follows from Eqs. (13) and (14) for the SCOP optical potential $V_{o p t}^{S C}(r)$,

$$
V_{o p t}^{S C}(r)=v_{01}(r) b_{010}^{\left(A_{1}\right)} S_{00}=v_{01}(r) / \sqrt{4 \pi} .
$$

As an example, Fig. 1 shows the SCOP potentials of propane for $T=100 \mathrm{eV}$. The radial Schrödinger equation, Eq. (5), was again solved using the SCOP potential $V_{o p t}^{\mathrm{SCOP}}(r)$ as the interaction potential to obtain the phase shifts. Here, the Schrödinger equation was solved by applying the variable phase approach (VPA) [47]. In this approach, the real part $\varepsilon_{l}(k r)$ and the imaginary part $\eta_{l}(k r)$ of the phase shift can be determined from the following two coupled differential equations:

$$
\begin{aligned}
& \varepsilon_{l}^{\prime}=-\frac{1}{k}\left[V_{R}^{S C}\left(X^{2}-Y^{2}\right)-2 V_{\mathrm{abs}}^{S C} X Y\right], \\
& \eta_{l}^{\prime}=-\frac{1}{k}\left[V_{R}^{S C}\left(X^{2}-Y^{2}\right)-2 V_{\mathrm{abs}}^{S C} X Y\right],
\end{aligned}
$$

with

$$
\begin{aligned}
& X=\cosh \eta_{l}\left(\hat{\eta}_{l} \sin \varepsilon_{l}-\hat{j}_{l} \cos \varepsilon_{l}\right), \\
& Y=\sinh \eta_{l}\left(\hat{\eta}_{l} \cos \varepsilon_{l}+\hat{j}_{l} \sin \varepsilon_{l}\right),
\end{aligned}
$$

where $\hat{j}_{l}$ and $\hat{\eta}_{l}$ are Riccati-Bessel functions. It should be noted that the original VPA formulas of Calogero [47] were transformed to Eq. (16) as the latter is more convenient and numerically stable.

The real and imaginary part of the phase shift $\varepsilon_{l}(k r)$ and $\eta_{l}(k r)$, respectively, at $r \rightarrow \infty$ are related to the scattering matrix $S_{l}$ via

$$
S_{l}(k)=\exp \left[2 i\left(\varepsilon_{l}+i \eta_{l}\right)\right]
$$


from which the integral elastic $\sigma_{e l}$, inelastic $\sigma_{i n e l}$, and TCS $\sigma_{t}$ can be derived via

$$
\begin{aligned}
\sigma_{e l}(k) & =\frac{\pi}{k^{2}} \sum_{l}(2 l+1)\left|1-S_{l}(k)\right|^{2}, \\
\sigma_{\text {inel }} & =\frac{\pi}{k^{2}} \sum_{l}(2 l+1)\left[1-\left|S_{l}(k)\right|^{2}\right], \\
\sigma_{t}(k) & =\frac{2 \pi}{k^{2}} \sum_{l}(2 l+1)\left[1-\operatorname{Re}_{l}(k)\right] .
\end{aligned}
$$

The phase shifts and the corresponding scattering matrix were obtained by the numerical integration of Eq. (16) using the fourth-order Runge-Kutta method [48]. The integration was performed until the relative change of the phase shifts for each $l$ was smaller than $1.0 \times 10^{-5}$.

\section{RESULTS AND DISCUSSION}

The experimental TCS and DCS of propane measured in this work are listed in Tables I and II, respectively. The uncertainties associated with the TCS are given in Table I. The relative uncertainty of the DCS amounted to $16 \%$. The uncertainties were determined according to the Guide to the Expression of Uncertainty in Measurement [49]. The description of the uncertainty analysis is not given here as it is detailed in other works of Baek et al. [4,30].

In Fig. 2, the TCS of propane measured in this work are compared to the results of other groups [8,9] who published their data in a tabular form. While the present results agree well with the measurements of Ariyasinghe et al. [9] within the experimental uncertainties, they are noticeably lower than the data of Szmytkowski and Kwitnewski [8], especially for $T<40 \mathrm{eV}$. A maximum at around $T=9 \mathrm{eV}$ can be found in both the present data and that of Szmytkowski and Kwitnewski [8]. Above $9 \mathrm{eV}$, the latter data decrease monotonically with increasing energy while the results of this work exhibit a shoulderlike structure at energies around $17 \mathrm{eV}$. A visual comparison of these data to that of Floeder et al. [5], Nishimura and Tawara [6], and Tanaka et al. [7], all of which were published in graphical forms, reveals that the present results agree with the data of Floeder et al. [5] and Tanaka et al. [7], whereas the results of Nishimura and Tawara [6] are close to those of Szmytkowski and Kwitnewski [8].

The present results for the DCS of propane are shown in Fig. 3 for six selected energies. They are compared to the experimental data of Boesten et al. [10] and of de Souza et al. [11]. The best agreement between the different results was found at $T=100 \mathrm{eV}$, where the data measured in this work agree with those of the other two groups over the whole angular range within the experimental uncertainties. At $T=40 \mathrm{eV}$ and $T=200 \mathrm{eV}$, the present data agree with the literature values for $\theta<90^{\circ}$. For $\theta \geqslant 90^{\circ}$, however, there is a significant difference between the present results and the literature values. The difference rises with increasing scattering angle. At $T=400 \mathrm{eV}$, the results of this work agree with those of de Souza et al. [11] for $\theta<60^{\circ}$, but are noticeably higher than the latter at larger scattering angles by as much as $60 \%$.

Figure 4 shows a comparison of the experimental DCS of propane obtained in this work with the theoretical values calculated using the IAM-SCAR and MIAM approach. As can be seen in Fig. 4, the IAM-SCAR method coarsely reproduces the present experimental results for electron energies down to $20 \mathrm{eV}$. At $T=20 \mathrm{eV}$, the IAM-SCAR approach agrees with the experimental results better than the MIAM approach. For $T>40 \mathrm{eV}$, however, the MIAM approach yields better agreement with the experimental values than the IAM-SCAR method. Here, the theoretical values calculated using the

\begin{tabular}{|c|c|c|c|c|c|}
\hline$T(\mathrm{eV})$ & $\sigma_{t}$ & $T(\mathrm{eV})$ & $\sigma_{t}$ & $T(\mathrm{eV})$ & $\sigma_{t}$ \\
\hline 3.8 & $26.79 \pm 0.42$ & 18 & $35.66 \pm 0.44$ & 46 & $30.76 \pm 0.39$ \\
\hline 4.8 & $31.74 \pm 0.50$ & 20 & $35.72 \pm 0.44$ & 50 & $29.68 \pm 0.37$ \\
\hline 5.8 & $38.09 \pm 0.59$ & 22 & $36.31 \pm 0.45$ & 60 & $27.16 \pm 0.34$ \\
\hline 6.9 & $42.03 \pm 0.53$ & 24 & $35.84 \pm 0.44$ & 70 & $24.89 \pm 0.31$ \\
\hline 7.8 & $43.32 \pm 0.54$ & 26 & $35.19 \pm 0.44$ & 80 & $23.75 \pm 0.31$ \\
\hline 8.9 & $43.71 \pm 0.54$ & 28 & $34.01 \pm 0.43$ & 90 & $22.93 \pm 0.28$ \\
\hline 9.9 & $41.39 \pm 0.52$ & 30 & $33.04 \pm 0.42$ & 100 & $21.81 \pm 0.27$ \\
\hline 12 & $38.77 \pm 0.48$ & 35 & $31.62 \pm 0.40$ & 110 & $20.72 \pm 0.26$ \\
\hline 14 & $37.22 \pm 0.47$ & 36 & $31.23 \pm 0.42$ & 120 & $19.98 \pm 0.25$ \\
\hline 16 & $36.29 \pm 0.48$ & 40 & $31.09 \pm 0.38$ & 140 & $18.47 \pm 0.23$ \\
\hline$T(\mathrm{eV})$ & $\sigma_{t}$ & $T(\mathrm{eV})$ & $\sigma_{t}$ & & \\
\hline 160 & $17.26 \pm 0.22$ & 600 & $7.62 \pm 0.10$ & & \\
\hline 180 & $16.49 \pm 0.21$ & 700 & $6.72 \pm 0.09$ & & \\
\hline 200 & $15.32 \pm 0.20$ & 800 & $5.91 \pm 0.08$ & & \\
\hline 220 & $14.13 \pm 0.18$ & 900 & $5.39 \pm 0.07$ & & \\
\hline 250 & $13.40 \pm 0.17$ & 1000 & $4.97 \pm 0.07$ & & \\
\hline 300 & $11.95 \pm 0.15$ & 1200 & $4.41 \pm 0.06$ & & \\
\hline 350 & $10.95 \pm 0.14$ & 1400 & $3.98 \pm 0.05$ & & \\
\hline 400 & $9.52 \pm 0.12$ & 1600 & $3.45 \pm 0.05$ & & \\
\hline 450 & $8.75 \pm 0.11$ & 1800 & $3.17 \pm 0.04$ & & \\
\hline 500 & $8.12 \pm 0.11$ & 2000 & $2.99 \pm 0.04$ & & \\
\hline
\end{tabular}
MIAM approach agree well with the experimental results

TABLE I. Total electron scattering cross sections (TCS) $\sigma_{t}$ of propane in units of $10^{-16} \mathrm{~cm}^{2}$. 
TABLE II. DCS $d \sigma_{e l} / d \Omega$ of propane in units of $10^{-16} \mathrm{~cm}^{2} / \mathrm{sr}$. The integral cross section $\sigma_{e l}$ and momentum transfer cross section $\sigma_{m t}$ are given in units of $10^{-16} \mathrm{~cm}^{2}$. The cross sections (italic font) at the scattering angles $0^{\circ}, 5^{\circ}, 150^{\circ}, 165^{\circ}$, and $180^{\circ}$ are extrapolated values obtained using the IAM-SCAR and MIAM approaches. The numbers in the square brackets are the powers of ten by which the preceding number is to be multiplied.

\begin{tabular}{|c|c|c|c|c|c|c|}
\hline$\theta / \mathrm{T}$ & $20 \mathrm{eV}$ & $30 \mathrm{eV}$ & $40 \mathrm{eV}$ & $60 \mathrm{eV}$ & $80 \mathrm{eV}$ & $100 \mathrm{eV}$ \\
\hline $0^{\circ}$ & 46.4 & 68.0 & 76.8 & 71.8 & 60.1 & 68.6 \\
\hline $5^{\circ}$ & 44.0 & 63.1 & 69.6 & 62.5 & 49.6 & 54.3 \\
\hline $10^{\circ}$ & 37.9 & 45.4 & 42.9 & 40.1 & 28.0 & 27.1 \\
\hline $15^{\circ}$ & 25.2 & 24.9 & 20.9 & 16.0 & 9.01 & 7.42 \\
\hline $20^{\circ}$ & 13.4 & 12.8 & 8.77 & 5.51 & 2.81 & 2.26 \\
\hline $25^{\circ}$ & 7.48 & 6.19 & 3.59 & 2.07 & 1.28 & 1.20 \\
\hline $35^{\circ}$ & 2.38 & 1.79 & 1.29 & 1.02 & $6.63[-1]$ & $6.01[-1]$ \\
\hline $45^{\circ}$ & 1.77 & 1.35 & $9.78[-1]$ & $6.28[-1]$ & $3.88[-1]$ & $3.48[-1]$ \\
\hline $60^{\circ}$ & 1.35 & $8.21[-1]$ & $5.27[-1]$ & $3.30[-1]$ & $2.40[-1]$ & $2.10[-1]$ \\
\hline $75^{\circ}$ & $9.23[-1]$ & $5.55[-1]$ & $3.47[-1]$ & $2.22[-1]$ & $1.19[-1]$ & $8.34[-2]$ \\
\hline $90^{\circ}$ & $7.68[-1]$ & $4.33[-1]$ & $3.08[-1]$ & $1.31[-1]$ & $8.17[-2]$ & $8.28[-2]$ \\
\hline $105^{\circ}$ & $7.98[-1]$ & $4.79[-1]$ & $3.18[-1]$ & $1.25[-1]$ & $1.18[-1]$ & $1.08[-1]$ \\
\hline $120^{\circ}$ & $9.45[-1]$ & $6.13[-1]$ & $4.25[-1]$ & $1.85[-1]$ & $1.58[-1]$ & $1.28[-1]$ \\
\hline $135^{\circ}$ & 1.20 & $8.19[-1]$ & $5.85[-1]$ & $2.75[-1]$ & $1.85[-1]$ & $1.86[-1]$ \\
\hline $150^{\circ}$ & 1.75 & 1.41 & $9.17[-1]$ & $3.42[-1]$ & $2.81[-1]$ & $1.87[-1]$ \\
\hline $165^{\circ}$ & 2.18 & 1.70 & 1.09 & $3.36[-1]$ & $3.83[-1]$ & $1.37[-1]$ \\
\hline $180^{\circ}$ & 2.34 & 1.81 & 1.15 & $3.26[-1]$ & $4.30[-1]$ & $1.04[-1]$ \\
\hline$\sigma_{e l}$ & 28.5 & 25.1 & 20.0 & 14.3 & 9.77 & 9.13 \\
\hline$\sigma_{m t}$ & 15.1 & 10.7 & 7.43 & 3.31 & 2.52 & 1.96 \\
\hline$\theta / \mathrm{T}$ & $200 \mathrm{eV}$ & $300 \mathrm{eV}$ & $400 \mathrm{eV}$ & $600 \mathrm{eV}$ & $800 \mathrm{eV}$ & $1000 \mathrm{eV}$ \\
\hline $0^{\circ}$ & 89.3 & 86.2 & 83.9 & 65.0 & 55.1 & 51.7 \\
\hline $5^{\circ}$ & 58.4 & 47.6 & 39.3 & 22.6 & 14.7 & 10.9 \\
\hline $10^{\circ}$ & 12.2 & 9.29 & 5.63 & 3.99 & 2.84 & 2.32 \\
\hline $15^{\circ}$ & 2.87 & 2.56 & 2.00 & 1.87 & 1.23 & $7.65[-1]$ \\
\hline $20^{\circ}$ & 1.43 & 1.52 & 1.12 & 1.10 & $6.43[-1]$ & $4.44[-1]$ \\
\hline $25^{\circ}$ & $9.14[-1]$ & $8.53[-1]$ & $6.36[-1]$ & $6.81[-1]$ & $3.47[-1]$ & $1.92[-1]$ \\
\hline $35^{\circ}$ & $4.07[-1]$ & $4.31[-1]$ & $2.74[-1]$ & $2.09[-1]$ & $1.12[-1]$ & $7.45[-2]$ \\
\hline $45^{\circ}$ & $2.47[-1]$ & $1.86[-1]$ & $1.49[-1]$ & $1.21[-1]$ & $5.24[-2]$ & $3.70[-2]$ \\
\hline $60^{\circ}$ & $9.05[-2]$ & $1.11[-2]$ & $7.77[-2]$ & $4.75[-2]$ & $2.36[-2]$ & $1.48[-2]$ \\
\hline $75^{\circ}$ & $7.36[-2]$ & $6.48[-2]$ & $3.92[-2]$ & $2.61[-2]$ & $1.31[-2]$ & $7.35[-3]$ \\
\hline $90^{\circ}$ & $5.89[-2]$ & $4.26[-2]$ & $2.62[-2]$ & $1.71[-2]$ & $7.96[-3]$ & $4.71[-3]$ \\
\hline $105^{\circ}$ & $4.51[-2]$ & $3.45[-2]$ & $1.98[-2]$ & $1.10[-2]$ & $5.13[-3]$ & $3.28[-3]$ \\
\hline $120^{\circ}$ & $4.60[-2]$ & $2.85[-2]$ & $1.60[-2]$ & $9.05[-3]$ & $4.00[-3]$ & $2.30[-3]$ \\
\hline $135^{\circ}$ & $4.88[-2]$ & $2.63[-2]$ & $1.21[-3]$ & $7.30[-3]$ & $3.23[-3]$ & $1.86[-3]$ \\
\hline $150^{\circ}$ & $4.83[-2]$ & $2.51[-2]$ & $1.20[-2]$ & $6.57[-3]$ & $2.98[-3]$ & $1.87[-3]$ \\
\hline $165^{\circ}$ & $4.16[-2]$ & $2.23[-2]$ & $1.19[.2]$ & $6.32[-3]$ & $2.56[-3]$ & $1.56[-3]$ \\
\hline $180^{\circ}$ & $3.51[-2]$ & $2.00[-2]$ & $1.15[-2]$ & $6.83[-3]$ & $2.94[-3]$ & $1.92[-3]$ \\
\hline$\sigma_{e l}$ & 6.04 & 5.04 & 3.75 & 2.60 & 1.60 & 1.14 \\
\hline$\sigma_{m t}$ & $8.49[-1]$ & $6.46[-1]$ & $4.06[-1]$ & $2.79[-1]$ & $1.37[-1]$ & $8.41[-2]$ \\
\hline
\end{tabular}

over the whole angular range, where they also reproduce the fine structures in the angular dependence of the DCS. Interestingly, the MIAM approach predicts a local maximum at about $\theta=150^{\circ}$ for electron energies around $100 \mathrm{eV}$, while a monotonic increase of the DCS with $\theta$ is expected according to the IAM-SCAR method. In view of the good agreement between the MIAM approach and the present experimental results, the MIAM approach was used to extrapolate the experimental DCS beyond the measured angular range, i.e., $\theta<10^{\circ}$ and $\theta>135^{\circ}$ for $T \geqslant 40 \mathrm{eV}$. For $T<40 \mathrm{eV}$, the IAM-SCAR method was applied for the extrapolation. Using the extrapolated experimental values, the integral elastic cross section $\sigma_{e l}$ and momentum transfer cross section $\sigma_{m t}$ were determined by the integration of $d \sigma_{e l} / d \Omega$ and $(1-\cos \theta) \times d \sigma_{e l} / d \Omega$, respectively, over the scattering angle. The values of $\sigma_{e x}$ and $\sigma_{m t}$ are given in Table II.

Figure 5 shows theoretical integral elastic $\sigma_{e l}$ and inelastic $\sigma_{\text {inel }}$ scattering cross sections of propane calculated using the SCOP model compared to the experimental values. In this work, the integral inelastic scattering cross sections were set equal to the integral ionization cross sections, which were taken from a recent work [4] of the authors. This complies with the calculation as the value of the excitation energy $\Delta$ in Eqs. (11) and (12) was set equal to the ionization potential of propane. As can be seen from Fig. 5, the SCOP model reproduces well the experimental integral elastic scattering cross sections for $T>30 \mathrm{eV}$. The good agreement between the theoretical and experimental values can also be seen in 


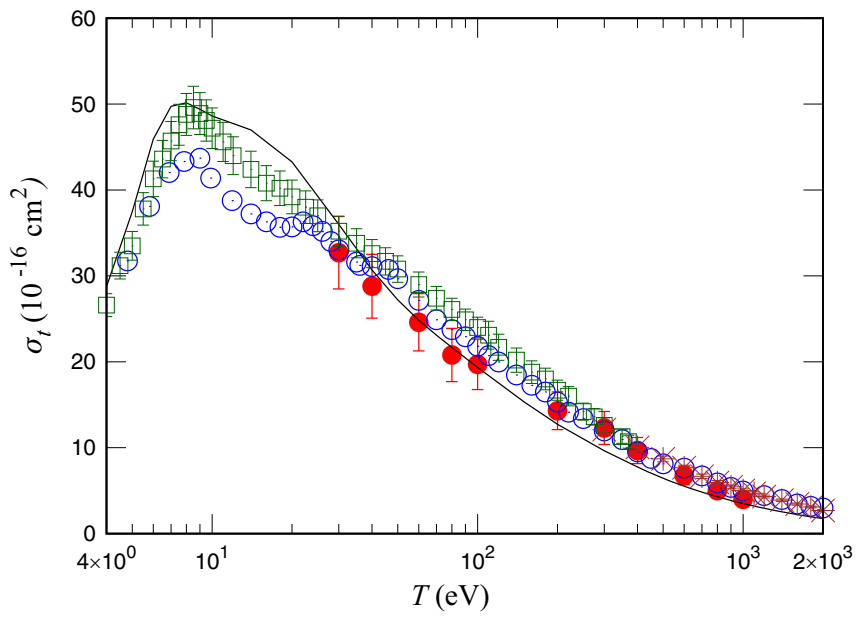

FIG. 2. Total electron scattering cross sections (TCS) $\sigma_{t}$ of propane measured by different groups and comparison of experimental to theoretical data: (O) present experimental results, $(\square)$ Szmytkowski and Kwitnewski [8], (*) Ariyasinghe et al. [9] (data only for $T>300 \mathrm{eV}$ ). The solid circle (๑) is the sum of experimental $\sigma_{e l}$ and $\sigma_{i n e l}$, and the solid line represents the theoretical results obtained using the SCOP model.

the case of integral inelastic scattering cross sections. It can also be seen from Fig. 5 that the SCOP model reproduces the experimental integral ionization cross sections over the whole energy range within the experimental uncertainties.

In Fig. 2, the theoretical TCS of propane calculated using the SCOP model are compared to those obtained experimentally. The theoretical values are noticeably higher than the experimental data of this work for $T \leqslant 40 \mathrm{eV}$, while a reasonably good agreement can be seen between the SCOP model and the results of Szmytkowski and Kwitnewski [8]. The largest difference between the SCOP model and the present experimental results occurred in the energy region from 10 to $20 \mathrm{eV}$, where the relative difference amounted to about $20 \%$. The position of the maximum TCS predicted by the SCOP model seems to be slightly lower than the experimental one. The difference in the position of the maximum may be caused by the overestimate of elastic scattering cross sections at low electron energies by the SCOP model and the neglection of the rotational as well as vibrational excitation cross sections in the theoretical calculations. As mentioned above, these excitation cross sections are included in the experimental data due to insufficient energy resolution of the electron energy analyzer.

For $T>40 \mathrm{eV}$, the theoretical TCS are noticeably lower than both the results of this work and those of Szmytkowski and Kwitnewski [8]. Here, the deviation between the theoretical and experimental TCS clearly exceeds the uncertainty associated with the latter. It can therefore be concluded that the SCOP model underestimates the scattering cross sections at high energies. However, the magnitude of this underestimation is on the order of the uncertainty of the sum of experimental $\sigma_{e l}$ and $\sigma_{\text {inel }}$, as can be seen in Fig. 2. Underestimation of TCS with the SCOP model for $T>100 \mathrm{eV}$ is only to a minor extent caused by the disregarding of the excitation cross sections in the calculation. The contribution of excitation cross sections to the TCS is expected to be minor for $T>100 \mathrm{eV}$ as ionization is the dominant inelastic collision process at high electron energies. Since not only the absolute value, but also the sign of the difference between the experimental and theoretical results changes with $T$, it may

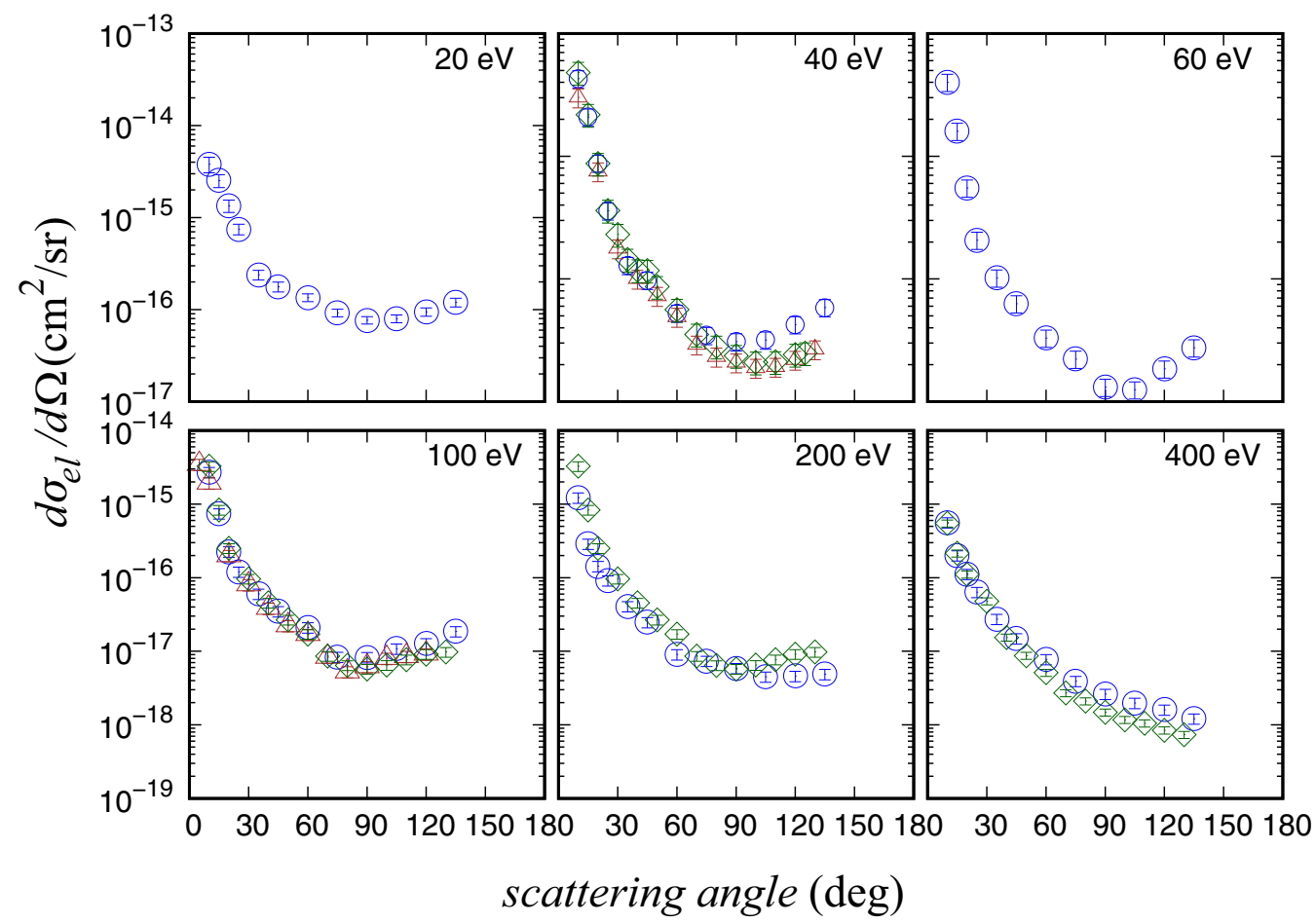

FIG. 3. Differential elastic electron scattering cross sections (DCS) $d \sigma_{e l} / d \Omega$ of propane for different electron energies as a function of scattering angle $\theta:(\bigcirc)$ present results, $(\triangle)$ Boesten et al. [10], $(\diamond)$ de Souza et al. [11] 


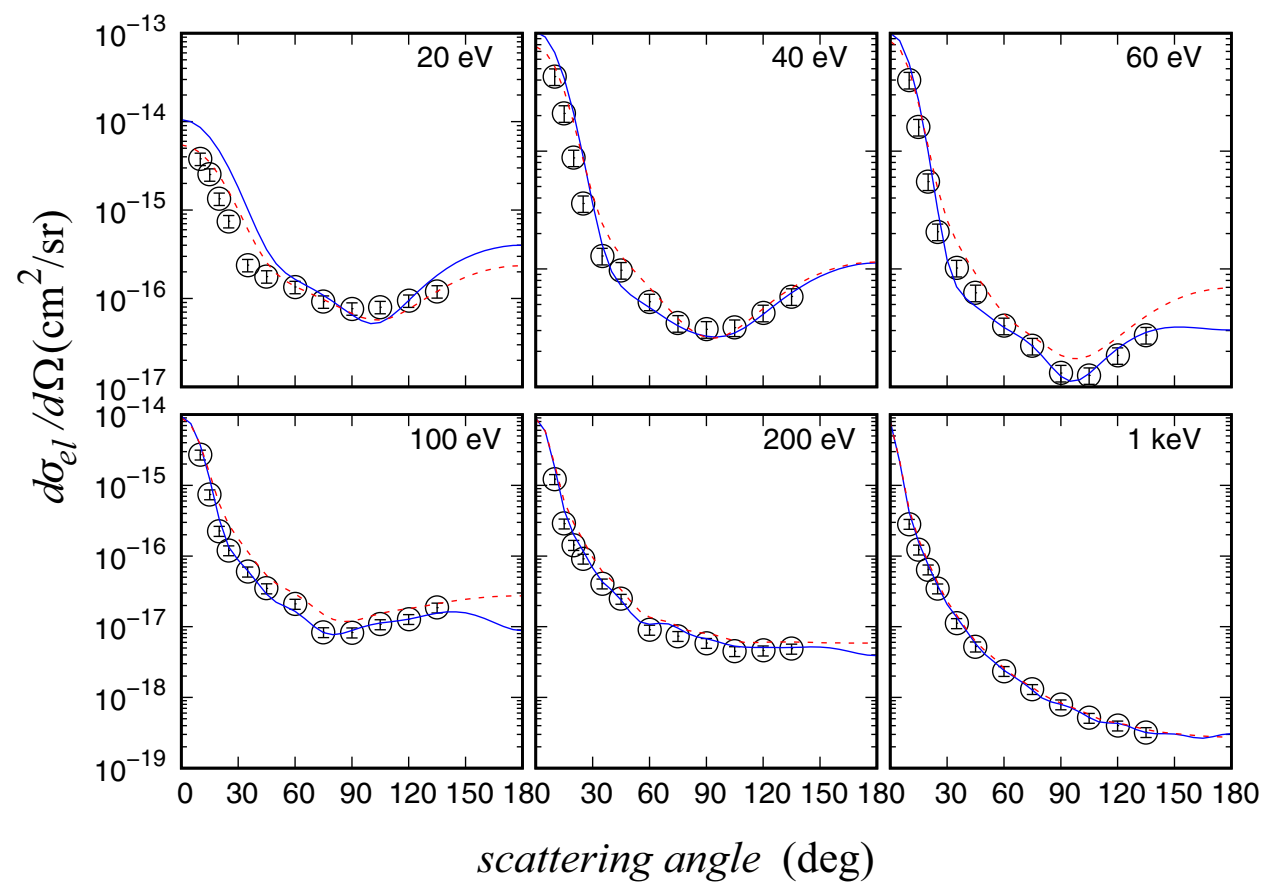

FIG. 4. Present experimental results for differential elastic electron scattering cross sections (DCS) $d \sigma_{e l} / d \Omega$ of propane (O) compared with calculated values using the IAM-SCAR (- - ) and the MIAM approach (-).

be appropriate to use an energy-dependent scaling factor for calculating the absorption potential $V_{\text {abs }}$ as discussed in the work of Staszewska et al. [50].

\section{CONCLUSION}

Total and elastic electron scattering cross sections of propane have been comprehensively measured over a broad energy and angular range. These data, together with the recently published doubly differential ionization cross sections of propane [4], provide a thorough description of the collision processes between electrons and propane. The electron

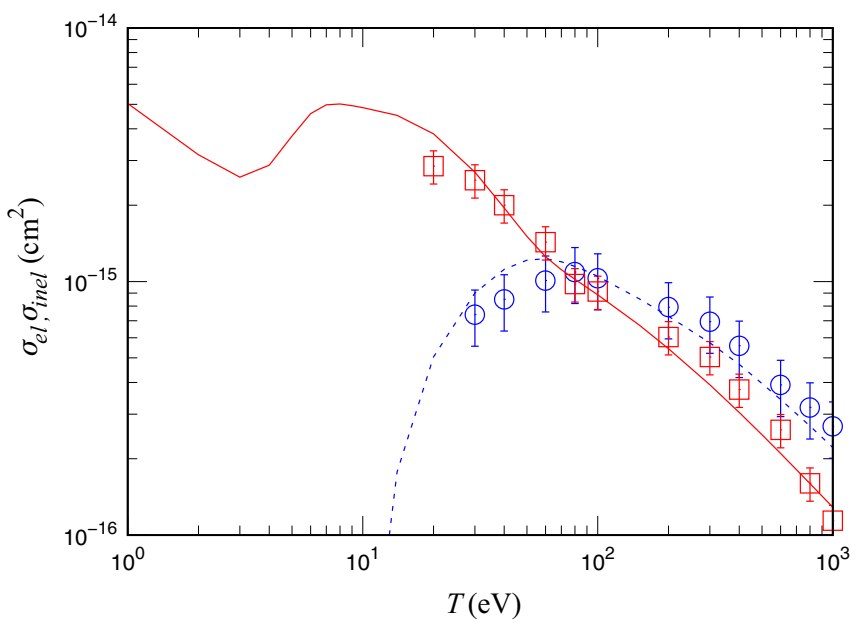

FIG. 5. Integral elastic $\sigma_{e l}(\bigcirc)$ and inelastic $\sigma_{\text {inel }}(\square)$ scattering cross sections [4] of propane. The solid and dashed line represent theoretical integral elastic and inelastic scattering cross sections, respectively. scattering by propane was also theoretically studied using $a b$ initio and semiempirical approaches. While the widely used IAM-SCAR approach was capable of coarsely reproducing the angular-dependent DCS over a wide energy range, the MIAM method appeared to be a more accurate approach for calculating the DCS of polyatomic molecules for $T>$ $40 \mathrm{eV}$. At these energies, good agreement between the MIAM method and the present results for the DCS of propane was observed. Even fine structures in the angular dependence of the DCS were reproduced by the MIAM method. It is interesting that the MIAM method predicted a local maximum at a scattering angle of about $150^{\circ}$ around $100 \mathrm{eV}$, while a monotonic angular dependence is here expected according to the IAM-SCAR approach. Measurements of the DCS of polyatomic molecules at scattering angles above $135^{\circ}$, for example, using the magnetic angle-changing technique, may be of interest from a theoretical point of view.

The experimental integral elastic and inelastic scattering cross sections of propane determined in this work could be reproduced by the SCOP model within uncertainties of about $16 \%$ and $25 \%$, respectively. Comparison of the sum of both theoretically calculated cross sections with rather accurately measured TCS, however, reveals that the SCOP model slightly underestimates the scattering cross sections for $T>50 \mathrm{eV}$.

\section{ACKNOWLEDGMENTS}

This work was carried out within the EMRP joint research project BioQuaRT. The EMRP was jointly funded by the EMRP participating countries within EURAMET and the European Union. The authors extend their thanks to Heike Nittmann for her assistance in conducting and evaluating the measurements. The technical assistance of Andreas Pausewang is also gratefully acknowledged. 
[1] International Atomic Energy Agency, ITER Technical Basis, ITER EDA Documentation Series No. 24 (IAEA, Vienna, 2002).

[2] H. G. Menzel, P. Pihet, and A. Wambersie, Int. J. Radiat. Biol. 57, 865 (1990).

[3] A. Bantsar, P. Colautti, V. Conte, G. Hilgers, M. Pietrzak, S. Pszona, H. Rabus, and A. Selva, Radiat. Prot. Dosim. 180, 177 (2018).

[4] W. Y. Baek, M. U. Bug, H. Nettelbeck, and H. Rabus, Eur. Phys. J. D 73, 61 (2019).

[5] K. Floeder, D. Fromme, W. Raith, A. Schwab, and G. Sinapius, J. Phys. B: At., Mol. Opt. Phys. 18, 3347 (1985).

[6] H. Nishimura and H. Tawara, J. Phys. B: At., Mol. Opt. Phys. 24, L363 (1991).

[7] H. Tanaka, Y. Tachibana, M. Kitajima, O. Sueoka, H. Takaki, A. Hamada, and M. Kimura, Phys. Rev. A 59, 2006 (1999).

[8] C. Szmytkowski and S. Kwitnewski, J. Phys. B: At., Mol. Opt. Phys. 35, 3781 (2002).

[9] W. M. Ariyasinghe, P. Wickramarachchi, and P. Palihawadana, Nucl. Instrum. Methods Phys. Res., Sect. B 259, 841 (2007).

[10] L. Boesten, M. A. Dillon, H. Tanaka, M. Kimura, and H. Sato, J. Phys. B 27, 1845 (1994).

[11] G. L. C. de Souza, M.-T. Lee, I. P. Sanches, P. Rawat, I. Iga, A. S. dos Santos, L. E. Machado, R. T. Sugohara, L. M. Brescansin, M. G. P. Homem, and R. R. Lucchese, Phys. Rev. A 82, 012709 (2010).

[12] B. Grosswendt and W. Y. Baek, Basic physical data in organic gases, in Radiation quality assessment based on physical radiation interaction at nanometer level, edited by P. Colautti, L. N. L.-I. N. F. N. Report No. 161 (Laboratori Nazionali di Legnaro, Legnaro, Italy, 2000).

[13] F. Blanco and G. García, Phys. Lett. A 330, 230 (2004).

[14] S. Hayashi and K. Kuchitsu, J. Phys. Soc. Jpn 41, 1724 (1976).

[15] A. Jain and S. S. Tayal, J. Phys. B: At. Mol. Phys. 15, L867 (1982).

[16] W. Y. Baek, M. Bug, H. Rabus, E. Gargioni, and B. Grosswendt, Phys. Rev. A 86, 032702 (2012).

[17] F. Blanco and G. García, Phys. Lett. A 317, 458 (2003).

[18] P. Limão-Vieira, M. Horie, H. Kato, M. Hoshino, F. Blanco, G. García, S. J. Buckman, and H. Tanaka, J. Chem. Phys. 135, 234309 (2011).

[19] H. Kato, A. Suga, M. Hoshino, F. Blanco, G. García, P. LimãoVieira, M. J. Brunger, and H. Tanaka, J. Chem. Phys. 136, 134313 (2012).

[20] H. Kato, K. Anzai, T. Ishihara, M. Hoshino, F. Blanco, G. García, P. Limão-Vieira, M. J. Brunger, S. J. Buckman, and H. Tanaka, J. Phys. B: At., Mol. Opt. Phys. 45, 095204 (2012).

[21] H. Murai, Y. Ishijima, T. Mitsumura, Y. Sakamoto, H. Kato, M. Hoshino, F. Blanco, G. García, P. Limão-Vieira, M. J. Brunger, S. J. Buckman, and H. Tanaka, J. Chem. Phys. 138, 054302 (2013).

[22] M. Hoshino, P. Limão-Vieira, K. Anzai, H. Kato, H. Cho, D. Mogi, T. Tanioka, F. Ferreira da Silva, D. Almeida, F. Blanco, G. García, O. Ingólfssson, and H. Tanaka, J. Chem. Phys. 141, 124302 (2014).
[23] A. G. Sanz, M. C. Fuss, F. Blanco, F. Carreli, F. Sebastianelli, F. A. Gianturco, and G. García, Appl. Radiat. Isot. 83, 68 (2014).

[24] D. F. Pastega, E. Lange, J. Ameixa, A. S. Barbosa, F. Blanco, G. García, M. H. F. Bettega, P. Limão-Vieira, and F. Ferreira da Silva, Phys. Rev. A 93, 032708 (2016).

[25] A. Jain, Phys. Rev. A 34, 3707 (1986).

[26] A. Jain, J. Chem. Phys. 86, 1289 (1987).

[27] A. Jain, J. Phys. B: At. Mol. Phys. 21, 905 (1988).

[28] A. Jain and K. L. Baluja, Phys. Rev. A 45, 202 (1992).

[29] W. Y. Baek, A. Arndt, M. U. Bug, H. Rabus, and M. Wang, Phys. Rev. A 88, 032702 (2013).

[30] W. Y. Baek and B. Grosswendt, J. Phys. B: At., Mol. Opt. Phys. 36, 731 (2003).

[31] Electron Source EQ 22/35, version 1 (Specs GmbH, Berlin, 2004).

[32] T. Edmonds and J. P. Hobson, J. Vac. Sci. Technol. 2, 182 (1965).

[33] M. J. Brunger and S. J. Buckman, Phys. Rep. 357, 215 (2002).

[34] H. S. W. Massey, E. H. S. Burhop, and H. B. Gilbody, Electronic and Ionic Impact Phenomena, Vol. 2 (Clarendon Press, Oxford, 1969).

[35] S. P. Khare, D. Raj, and P. Sinha, J. Phys. B: At., Mol. Opt. Phys. 27, 2569 (1994).

[36] F. Salvat, A. Jablonski, and C. J. Powell, Comput. Phys. Commun. 165, 157 (2005).

[37] J. B. Furness and I. E. McCarthy, J. Phys. B: At. Mol. Phys. 6, 2280 (1973).

[38] B. H. Bransden and C. J. Joachain, Physics of Atoms and Molecules (Longman, New York, 1983).

[39] A. Jain, J. Phys. B: At. Mol. Phys. 15, 1533 (1982).

[40] GAUSSIAN09, Gaussian, Inc., Wallingford, CT.

[41] S. Salvini and D. G. Thompson, J. Phys. B: At. Mol. Phys. 14, 3797 (1981).

[42] F. A. Gianturco and J. A. Rodriguez-Ruiz, J. Mol. Struct. 260, 99 (1992).

[43] J. P. Perdew and A. Zunger, Phys. Rev. B 23, 5048 (1981).

[44] N. Sanna and F. A. Gianturco, Comput. Phys. Commun. 128, 139 (2000).

[45] G. Staszewska, D. W. Schwenke, D. Thirumalai, and D. G. Truhlar, Phys. Rev. A 28, 2740 (1983).

[46] S. G. Lias, Ionization energy evaluation, in NIST Chemistry WebBook, NIST Standard Reference Database No. 69, edited by P. J. Linstrom and W. G. Mallard (National Institute of Standards and Technology, Gaithersburg, MD, 20899).

[47] F. Calogero, Variable Phase Approach to Potential Scattering (Academic Press, New York, 1967).

[48] J. D. Lambert, Numerical Methods for Ordinary Differential Systems: The Initial Value Problem (Wiley, New York, 1991).

[49] International Organization for Standardization (ISO), Guide to the Expression of Uncertainty in Measurement (ISO, Geneva, 1993).

[50] G. Staszewska, P. Staszewski, and K. Żebrowski, J. Electron. Spectrosc. Relat. Phenom. 162, 56 (2008). 